\title{
Measuring The Tensile Strain of Wood By Visible And Near-Infrared Spatially Resolved Spectroscopy
}

Ma Te ( $\square$ mate@agr.nagoya-u.ac.jp )

Nagoya Daigaku Daigakuin Seimei Nogaku Kenkyuka Nogakubu

Tetsuya Inagaki

Nagoya University: Nagoya Daigaku

Masato Yoshida

Nagoya University: Nagoya Daigaku

Mayumi Ichino

Nagoya University: Nagoya Daigaku

Satoru Tsuchikawa

Nagoya University: Nagoya Daigaku

\section{Research Article}

Keywords: Tensile strain of wood, non-destructive evaluation, spatially resolved spectroscopy, visible and short-wave light scattering, multivariate analysis

Posted Date: June 17th, 2021

DOl: https://doi.org/10.21203/rs.3.rs-570550/v1

License: (c) (i) This work is licensed under a Creative Commons Attribution 4.0 International License.

Read Full License 
1 Measuring the Tensile Strain of Wood by Visible and Near-

2 Infrared Spatially Resolved Spectroscopy

3

4 Te Ma, Tetsuya Inagaki, Masato Yoshida, Mayumi Ichino, and Satoru Tsuchikawa*

6 *Corresponding author: Satoru Tsuchikawa, Graduate School of Bioagricultural

7 Sciences, Nagoya University, Furo-Cho, Chikusa, Nagoya 464-8601, Japan, Email

$8 \quad$ addresses: $\underline{\text { st3842@agr.nagoya-u.ac.jp }}$

9 Te Ma (mate@agr.nagoya-u.ac.jp), Tetsuya Inagaki (inatetsu@agr.nagoya-u.ac.jp),

10 Masato Yoshida (yoshida@agr.nagoya-u.ac.jp), and Mayumi Ichino

11 (ichino.mayumi@j.mbox.nagoya-u.ac.jp): Graduate School of Bioagricultural

12 Sciences, Nagoya University, Furo-Cho, Chikusa, Nagoya 464-8601, Japan.

14 Declarations:

15 Funding: This study is supportted by JSPS (KAKENHI, no.19K15886)

16 Conflicts of interest/Competing interests: Not applicable

17 Availability of data and material: Yes

18 Code availability: Not applicable 
19 Authors' contributions:

20 Te Ma: methodology, data collection and analysis, writing-original draft preparation

21 Tetsuya Inagaki: methodology, data validation, and writing- reviewing.

22 Masato Yoshida: contributed to capture the submicroscopic changes during tension

23 testing, writing- reviewing

24 Mayumi Ichino: data collection and analysis

25 Satoru Tsuchikawa*: supervision, conceptualization, writing- reviewing and editing

26 Ehics approval: Not applicable

27 Consent to participate: Not applicable

28 Consent for publication: Not applicable

30 Abstract:

Wood has various mechanical properties, so stiffness evaluation is critical for quality management. Using conventional strain gauges constantly is high cost, also challenging to measure precious wood materials due to the use of strong adhesive. This study

34 demonstrates the correlation between light scattering changes inside the wood cell walls and tensile strain. A multifiber-based visible-near-infrared (Vis-NIR) spatially resolved spectroscopy (SRS) system was designed to rapidly and conventiently acquire such light 
scattering changes. For the preliminary experiment, samples with different thicknesses were measured to evaluate the influence of thickness. The differences in Vis-NIR SRS spectral data diminish with an increase in sample thickness, which suggests that the SRS method can successfully measure the whole strain (i.e., surface and inside) of wood samples. Then, for the primary experiment, 18 wood samples with the same thickness $(2$ characterized by a determination coefficient $\left(R^{2}\right)$ of 0.86 with a root mean squared error (RMSE) of $297.89 \mu \varepsilon$ for five-fold cross-validation; for test validation, The prediction accuracy was characterized by an $R^{2}$ of 0.82 and an RMSE of $345.44 \mu \varepsilon$.

\section{Keywords:}

Tensile strain of wood, non-destructive evaluation, spatially resolved spectroscopy, visible and short-wave light scattering, multivariate analysis

\section{Introduction}

Wood is a natural material with multi-layered elongated cells. Due to the variability of its mechanical properties, the stiffness evaluation of each structural wooden member

54 is critical for quality management (Baillères et al. 2012; Montero et al. 2012). The wood 
cell wall is a macromolecular composite formed of cellulose, hemicelluloses, and lignin

56 (Hon and Chang 1984). Cellulose is the primary component in bearing tensile stress

57 (Salmén and Bergström 2009). In contrast, hemicelluloses function as a coupling agent to hold the cellulose (Burgert 2006). The conventional method for wood strain measurement is to use a strain gauge, which is high cost (either disposable or reusable ones) in constant use (Yang et al. 2005). In addition, it is challenging to measure precious

61 wood materials due to the use of a strong adhesive, which can destroy the wood after removal. For example, the heritage community generally does not allow the application of strain gauges on wooden arts (Anaf et al. 2020). Moreover, difficulties arise when strain gauges are used in an environment where the electromagnetic wave interference is extensive (Liu et al. 2015; Barr et al. 2017). and Salmén 2001) can detect wood structure-function relationships at the nano- and

69 microstructural levels. Indeed, Raman spectroscopy (Hsieh et al. 2008) can predict sample strains based on a typical shift peak at a wavenumber of $1095 \mathrm{~cm}^{-1}$, which corresponds to the glycosicdic bond in the cellulose structure backbone (Guo and Altaner 2018). Moreover, the band shift varies among specimens (Eichhorn 2001), and the 
molecular strain is smaller than the macroscopic strain (Peura et al. 2007). In contrast,

74 Near-IR (NIR) spectroscopy (wavelength: $800-2500 \mathrm{~nm}$ or wavenumber: $12500-4000$ $\mathrm{cm}^{-1}$ ) is well-suited for characterizing organic compounds in samples, mainly in combination with multivariate mathematical techniques. When NIR light illuminates and transmits through an object, the energy of the incident electromagnetic wave changes due to the stretching and bending vibrations of chemical bonds, such as $\mathrm{O}-\mathrm{H}, \mathrm{N}-\mathrm{H}$, and $\mathrm{C}-$ H. Subsequently, the quality and quantity of an object can be evaluated nondestructively, rapidly, and cost-effectively by analyzing the light reflectance and transmittance values (Ma et al. 2020). Compared with microtomed sections needed for IR spectroscopy, NIR spectroscopy can nondestructively measure wood samples up to several millimeter thick without special sample pretreatments (Salmén and Bergström 2009). This is essential when focusing on practical applications, as thin samples prepared in lab behave differently than solid wood, e.g., including stress relaxation in the former (Yu et al. 2009).

Taking advantages of NIR spectroscopy, Guo et al. (2019) analyzed band shifts and band assignments on NIR light absorbance characteristics during the wood tension test, the results of which suggest that the observed band shifts correlate with wood tension levels. Their study is impressive and has reference value for future use of NIR spectroscopy.

90 However, several issues can be highlighted, such as small-signal band shifts on the 
91 collected NIR spectra; e.g., a sample strain of $0.1 \%$ would induce a band shift of 92 approximately $2 \mathrm{~cm}^{-1}$ at around $6470 \mathrm{~cm}^{-1}(3 \mathrm{OH} \cdots 5 \mathrm{O})$. Moreover, since the molecules 93 are influenced by neighboring molecules, actuarial peaks overlap on the NIR spectra 94 (Okazaki 2012). Indeed, advanced curve fitting approaches could be used to predict the 95 peak shift, but the band shift varies among specimens, which has been confirmed by the 96 IR method (Eichhorn 2001). The spectral information of such long-wave sensitive 97 spectrometers is rather rich, and, as such, it requires expensive equipment, such as detectors and light sources (Xing et al. 2008). Accordingly, there is still room to develop and improve NIR spectra collection and data analyisis methods, expecially for practical application purposes . The most likely to be neglected is that bulk wood is a highly scattering medium, and the light scattering degree inside the wood cell wall also highly correlates with the microstructur (Ban et al. 2018; Ma et al. 2018a, 2019). Studies show that the reduced light scattering coefficient $\left(\mu_{s}^{\prime}=10-100 \mathrm{~cm}^{-1}\right)$ is much larger than the absorption 106 coefficient $\left(\mu_{a}=0.05-1.00 \mathrm{~cm}^{-1}\right)$ in the wavelength range of 700-1040 $\mathrm{nm}$ for both softwood and hardwood species treated in different ways (dry, wet and degraded)

108 (D'Andrea et al. 2007). The deformation under longitudinal tension includes 
macromolecule deformations in the layers and interlaminar slippages. The former is

110 related to the structure, orientation, and interaction of the polymers in the wood, and the

111 latter slippage deformation results from the structural differences between cell-wall layers

112 (Keckes et al. 2003; Adler and Buehler 2013). The misalignment between the cellulose

113 fibrils to the strain direction is amplified by bending and shearing of anatomical structures

114 and straightening of slightly waved cellulose microfibrils, forming lenticular spaces

115 between adjacent fibrils (Salmén 2015). The weak interfaces of wood cells or annual rings

116 could deflect transverse cracks into the longitudinal plane (Smith et al. 2003; Marthin and

117 Kristofer Gamstedt 2019; Guo et al. 2020). Hence, effective utilization of the light

118 scattering degrees (i.e., microstructure changs) inside the wood during tension testing

119 should predict strain levels accurately. This method also can reduce costs associated with

120 equipment because shorter wavelengths are scattered more strongly than longer

121 wavelengths in the visible (Vis)-NIR optical range (Ma et al. 2018a).

However, conventional Vis-NIR spectrometry acquires spectral data from a single sample point based on the collective effects of light absorption (due to chemical components such as water and cellulose content) and scattering (due to physical structures such as cell size and intercellular spacing) (Vanoli et al. 2020). Studies have mainly relied 
127 on performing further spectral pretreatments, such as baseline offset correction or 128 standard normal variate (SNV), that reduce light scattering effects before training 129 calibration models (Zude et al. 2011). Spatially resolved spectroscopy (SRS) requires 130 relatively strong, steady-state spotlights for illumination, and its diffusely reflected light 131 pattern is collected at multiple distances for light absorption and scattering evaluation 132 (Farrell et al. 1992; Qin et al. 2009; Lu et al. 2020). SRS has two main measurement 133 configurations: spectral imaging and fiber probing. On the one hand, the SRS based on 134 spectral imaging is a non-contact method that measures spatially resolved diffuse 135 reflectance over a broad spectral range (Peng and Lu 2008; Qin and Lu 2008; Zhu et al. 2015). The measurement system mainly consists of a hyperspectral imaging (HSI) camera,

137 a prime lens, and a small broadband beam as illumination. However, the light beam and source-detector distance need to be carefully considered in this configuration, as they 139 determine the measured results (Cen and Lu 2010; Lu et al. 2020). On the other hand, the 140 fiber probe-based SRS is a contact method, which is often inconvenient for rapid online 141 quality assessment (Ma et al. 2018a). However, due to the easy-to-operate design with a 142 strong light reflectance, fiber probe-based SRS portable systems are desirable alternatives 143 for on-site applications. Besides, contact methods are more suitable to predict the strains 144 of moving wood sampels caused by tension testing than non-contact methods. 
146 in Vis-NIR SRS spectral data collected from wood samples during tension testing. The

147 objectives of this paper are as follows: (1) design a multifiber-based Vis-NIR SRS system

148 to rapidly and nondestructively acquire light scattering characteristics; (2) examine the

149 relationship between SRS signals and wood tension strains by principal component analysis (PCA); and (3) construct wood strain calibration models by partial least squares

151 (PLS) regression. This study should provide new insights into SRS methods for practical application to predict the tensile strain of wood conveniently and cost-effectively.

\section{Materials and methods}

\subsection{Sample preparation}

Wood samples (Hinoki cypress) with a length of $120 \mathrm{~mm}$ (longitudinal), a width of 10 $\mathrm{mm}$ (radial), and various thicknesses (tangential: $2 \mathrm{~mm}, 3 \mathrm{~mm}, 4 \mathrm{~mm}$, and $5 \mathrm{~mm}$ ) were sawn from air-dried wood board commercially purchased from a local wood processing company. Specimens were selected from mature wood parts sufficiently far from the pith to neglect ring curvature. 
163 primary experiment, 18 wood samples with the same thickness $(2 \mathrm{~mm})$ were tested to

164 construct a strain calibration model. The samples were selected based on the wood fiber

165 directions, which were as parallel as possible to the longitudinal direction. Before the

166 experiment, all the samples remained in a desiccator, where relative humidity (RH) was

167 controlled at $59 \%$ with a saturated salt solution of sodium bromide. Subsequently, the

168 sample weights were measured for the equilibrated specimens using a digital balance

169 (accuracy of $0.0001 \mathrm{~g}$ ). A digital caliper (0.01 mm accuracy) measured the sample

170 dimensions under the equilibrated condition. From the measured weights and dimensions

171 of the raw data, sample equilibrium moisture content (EMC) and density were calculated according to the following equations: respectively, and $V$ is the sample volume under the equilibrated condition.

\subsection{Tensile test}

Each prepared wood sample was placed in a bending testing machine (preliminary 
181 50NA, IMADA-SS Corporation, Japan). During tension test, the bending machine was

182 suspended several times manually to obtain strain measurements and Vis-NIR SRS data.

183 The strain was recorded with strain gauges (FLAB-5-11, Tokyo Sokki Kenkyujo, Japan)

184 glued to one side of each sample with instant adhesive (CN, Tokyo Sokki Kenkyujo,

185 Japan) and connected to a strain-meter (TC-32K, Tokyo Sokki Kenkyujo, Japan). A Vis-

186 NIR measurement system was used to collect light scattering characteristics on the other

187 sample side (Fig. 1 (a) and (b)).

188

\subsection{Visible and near-infrared spatially resolved spectroscopy measurements}

Fig. 1 (c) and (d) show the measurement part (i.e. the fixator of light illumination and detection fibers) of the proposed Vis-NIR SRS system and a diagram of the internal structure. A 5-W halogen lamp initially provided light illumination. An optical fiber (SOG-70S, Sumita Optical Glass, Inc., Saitama, Japan) translated the light source onto each wood sample. Then, 30 silica fibers (Vis-NIR type, Core: $100 \mu \mathrm{m}$, Clad: $110 \mu \mathrm{m}$,

195 Fiberguide Industries, New Jersey, USA) were separated into five groups (1, 2, 3, 4, and

1965 from the light illumination point) to collect the diffuse reflected light and transfer it to the Vis-NIR HSI camera (SPECT-100nir1F, Spectral Application Research Laboratory

198 Co., Ltd. Shizuoka, JAPAN). Inside the camera, the light beam was dispersed into spectral 
components (vertical axis) while preserving spatial information (horizontal axis), and the camera took two-dimensional light signals. The shutter speed and framerate were set at

$20115 \mathrm{~ms}$ and $8 \mathrm{fps}$, respectively. In this study, the fixator was pasted parallelly to the sample

202 grain direction with a double-sided tape, which was easily removable after measurement

203 collection. The sensitive wavelength range of the Vis-NIR HSI camera was 600-1100

$204 \mathrm{~nm}$, with a minimum reading width of approximately $0.65 \mathrm{~nm} /$ pixel and a spectral

205 resolution of $4.5 \mathrm{~nm}$. For each measurement, 16 spectral images were captured and their

206 averaged values saved. Light reference was measured using a tailor-made integrating

207 sphere, i.e., a plastic ball (diameter of $6 \mathrm{~cm}$ ) covered in barium sulfate. Dark values were

208 measured by turning off the light and covering the light-collection fibers. Wavelengths

209 under $660 \mathrm{~nm}$ and over $1002 \mathrm{~nm}$ were noisy and unreliable. Thus, a wavelength range of

$210 \quad 660-1002 \mathrm{~nm}$ was selected for further data analysis. The collected spectra were then

211 converted to the reflectance values by Eq. (3):

$$
R_{\lambda}=\frac{s_{\lambda}-D_{\lambda}}{B_{\lambda}-D_{\lambda}}
$$

213 where $\lambda$ denotes the wavelength, $S$ and $B$ are the sample and a white reference spectrum,

214 respectively, and $D$ is the dark spectrum. 
216 (thickness of $2 \mathrm{~mm}$ ) before and after the tensile test to understand the submicroscopic

217 changes during tension testing.

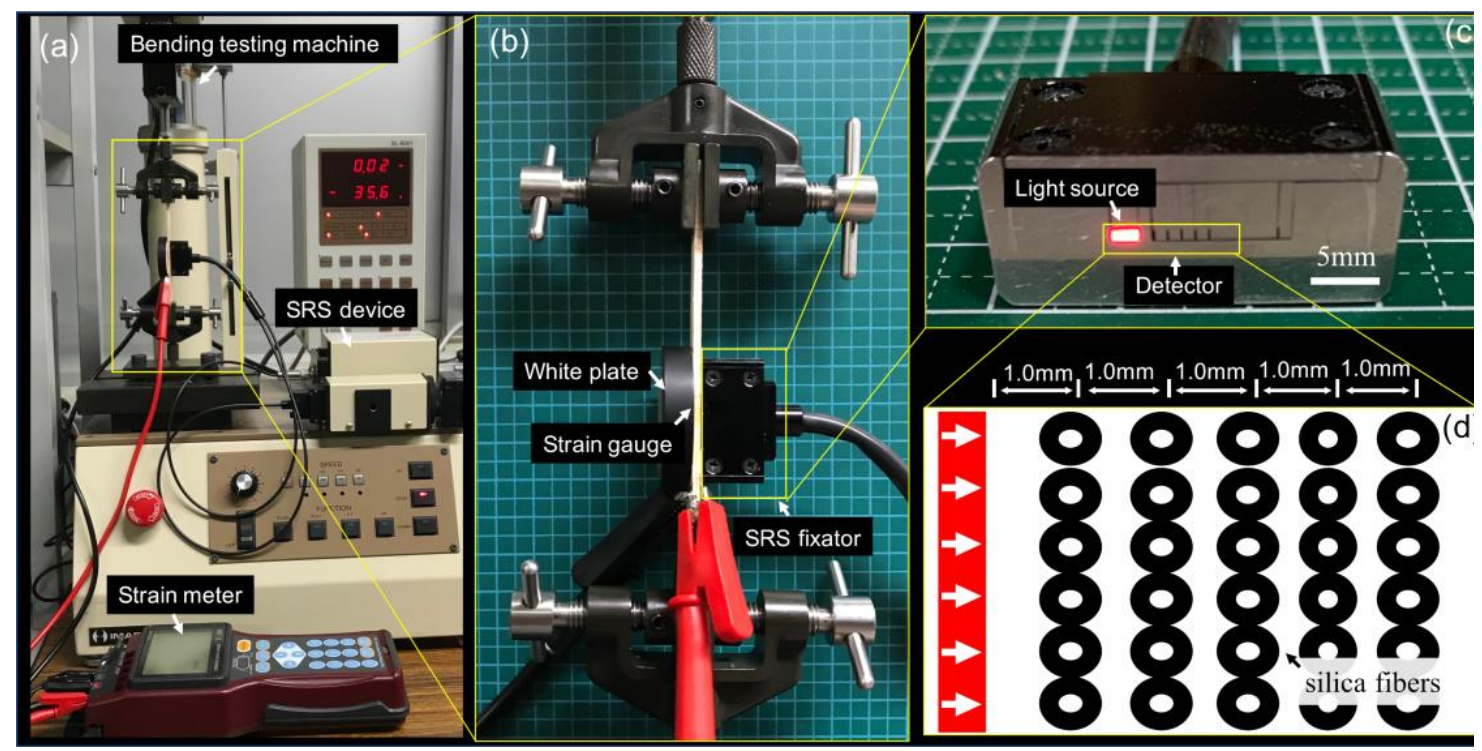

Fig. 1 (a) Experiment instruments; (b) Vis-NIR SRS data and strain measurements; (c) fixator of the Vis-NIR SRS measurement system; (d) internal structure diagram of the fixator.

\subsection{Spectra pretreatments and principal component analysis}

order: 2; frame length: 15). The spectral data, collected before tension testing subtracted 
226 (e.g., SNV (Cuesta Sánchez et al. 1995) and the second derivative (Gorry 1991)) were

227 used in this study to keep the maximum light scattering information.

\subsection{Partial least squares regression analysis}

The Vis-NIR difference spectral data were calibrated with the measured strain values

via PLS regression (Martens and Tormod 1992). In developing the PLS regression models, the constructed calibration model's performance:

$$
\begin{gathered}
R^{2}=1-\frac{\sum_{i=1}^{n}\left(y_{i}-\hat{y}_{i}\right)^{2}}{\sum_{i=1}^{n}\left(y_{i}-\bar{y}\right)^{2}} \\
\text { RMSE }=\sqrt{\frac{1}{n} \sum_{i=1}^{n}\left(\hat{y}_{i}-y_{i}\right)^{2}}
\end{gathered}
$$

where $n$ is the number of measurements, $y$ is the reference strain values, $\hat{y}$ is the strain value predicted by PLS regression analysis, and $\bar{y}$ is the mean value of $y$. the SRS spectral data collected by the individual fiber groups on the PLS model (Wold et Inc., Natick, MA). 


\section{Results and discussion}

Fig. 2 shows the raw spectral image of a wood sample with a thickness of $2 \mathrm{~mm}$ taken

247 by the Vis-NIR HSI camera before the tensile test. This image data contains the spatial

248 information of the 30 silica fibers (horizontal axis) and the spectral information of the

249 measured wood sample (vertical axis). The main difficulty with conventional SRS

250 methods is associated with collecting the spectral data with a high signal-to-noise ratio

251 quickly. One method involves repeating the same data measurements and averaging the

252 results, which is time-consuming (Tkachenko 2006). This study is desirable for the spectral data acquisition time to be short of excluding additional relaxation phenomena

254 (Burgert 2006; Altaner et al. 2014) This was achieved by a two-step signal averaging process: (i) each fiber occupies 34 pixels of the HSI camera, and the central 30 pixels were averaged for spectral data collection, after which (ii) the signals of six fibers in the same group were averaged. 


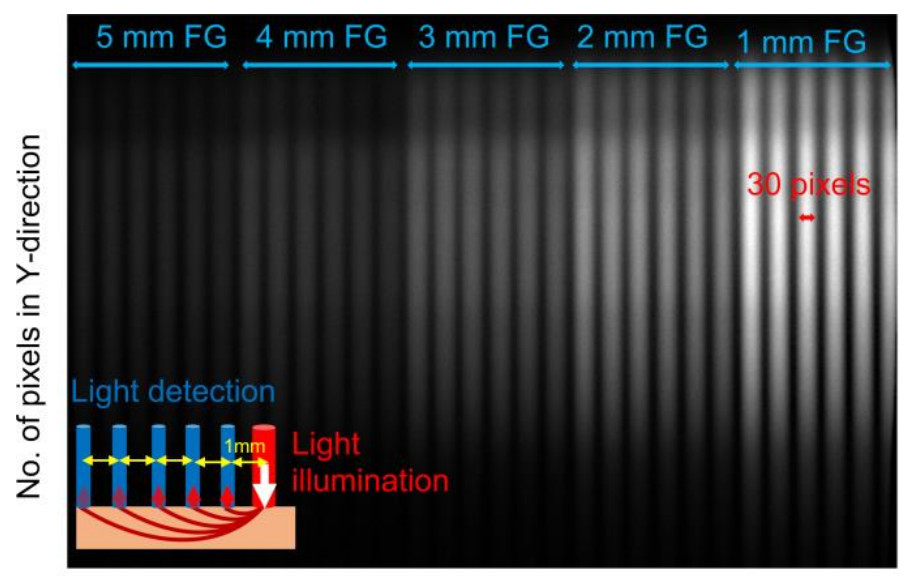

No. of pixels in X-direction

Fig. 2. Raw Vis-NIR SRS spectral image of wood sample with $2 \mathrm{~mm}$ thickness.

Fig. 3 shows the Vis-NIR SRS spectra with standard deviations of the 18 wood samples at various tension levels, which were initially calculated from the raw spectral image shown in Fig. 2. It is logical that the overall spectral intensity quickly falls with an increase in distance from the light illumination. The wavelength at $925 \mathrm{~nm}$ corresponds to the third overtone of $\mathrm{C}-\mathrm{H}$ absorption (Mohammadi-Moghaddam et al. 2018), which can be attributed to the chemical components of the wood samples. The wavelength at approximately $930 \mathrm{~nm}$ has the highest light reflectance when the fiber group is $3-4 \mathrm{~mm}$ away from the light illumination, suggesting that the light at said wavelength was less absorbed and transmittted further from the light illumination than other wavelengths along the wood grain direction. It is noteworthy that the optical scattering was not isotropic within each wood sample; the light propagated further in the parallel direction 
270 because the scattering coefficient along the cylinders is much smaller than that in the

271 perpendicular direction to the cylinders (Ma et al. 2018b, 2019).

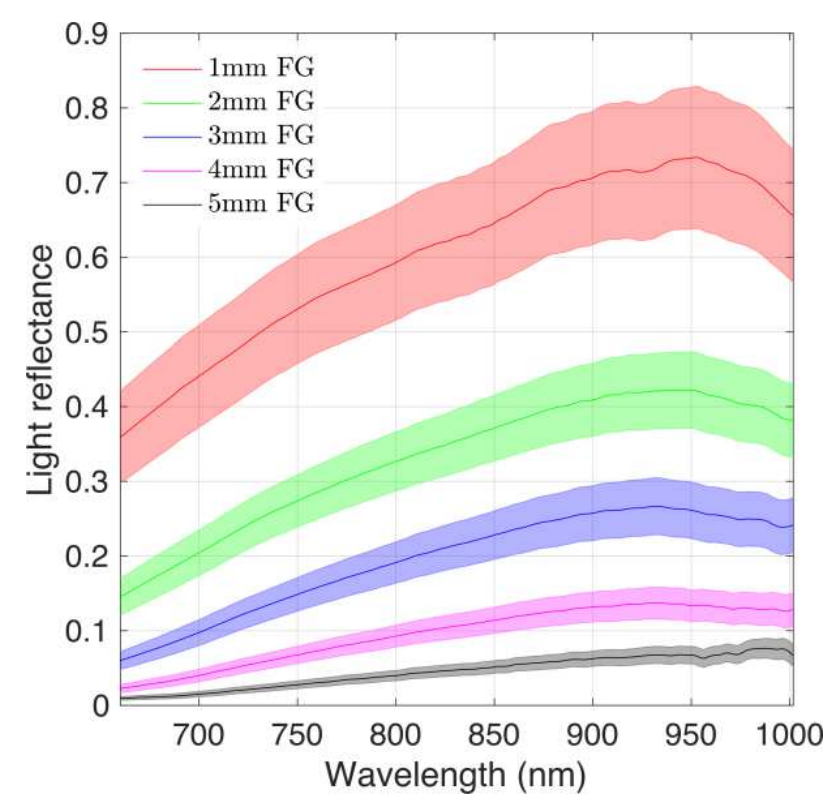

Fig. 3 Averaged Vis-NIR raw spectra and the standard deviations (light color) of 18 samples with the same thickness $(2 \mathrm{~mm})$ and various tension strains.

Fig. 4 shows the Vis-NIR spectral data for various strain measurements for wood

274 samples with different thicknesses (vertical) by the five fiber groups (horizontal),

275 respectively. The wavelength range was selected to $900-950 \mathrm{~nm}$ to expand the image size.

276 The light reflectance increased with an increase in wood strain. Light absorption at 925

$277 \mathrm{~nm}$ is the most obvious at the spectra collected by the 1-mm fiber group. The signal

278 quality decreases with an increase in distance between the light illumination and light-

279 detection fibers, suggesting that different fiber groups can collect spectral data with 
280 different light absorption and scattering degrees. Light reflectance is also affected by

281 sample thickness. The differences in the Vis-NIR spectral data, caused by sample strains,

282 diminished in thicker samples, especially at far fiber groups. This could be due to the light

283 transmission depth is different among the wood samples at various thicknesses. In this

284 study, the Vis-NIR light was transmitted through hinoki wood samples with an

285 approximate thickness of $5 \mathrm{~mm}$ (see the supplementary, Fig. S1), after which it was

286 reflected by the white plate and detected by optical fibers. However, because thicker wood

287 samples have a more profound light transmission, which affectted light propagating in

288 parallel, stronger noise was associated with the collected spectra at more extended fiber

289 groups. Except for signal quality, because the strain gauge was stuck on the opposite side

290 of the SRS fixator, less transmission light could also reduce the correlation between SRS

291 data and strain reference values. This suggests that the proposed SRS equipment is

292 suitable for predicting the tension strain of wood sampels with an approximately of 2-3

293 mm. Further improvements could be useful to construct strain prediction models for

294 thicker samples, such as reducing the distance between fiber groups and introducing a

295 method to measure the strain changs where the spectral data were collected. 
Fiber group from 1-mm to 5-mm (1-mm interval)

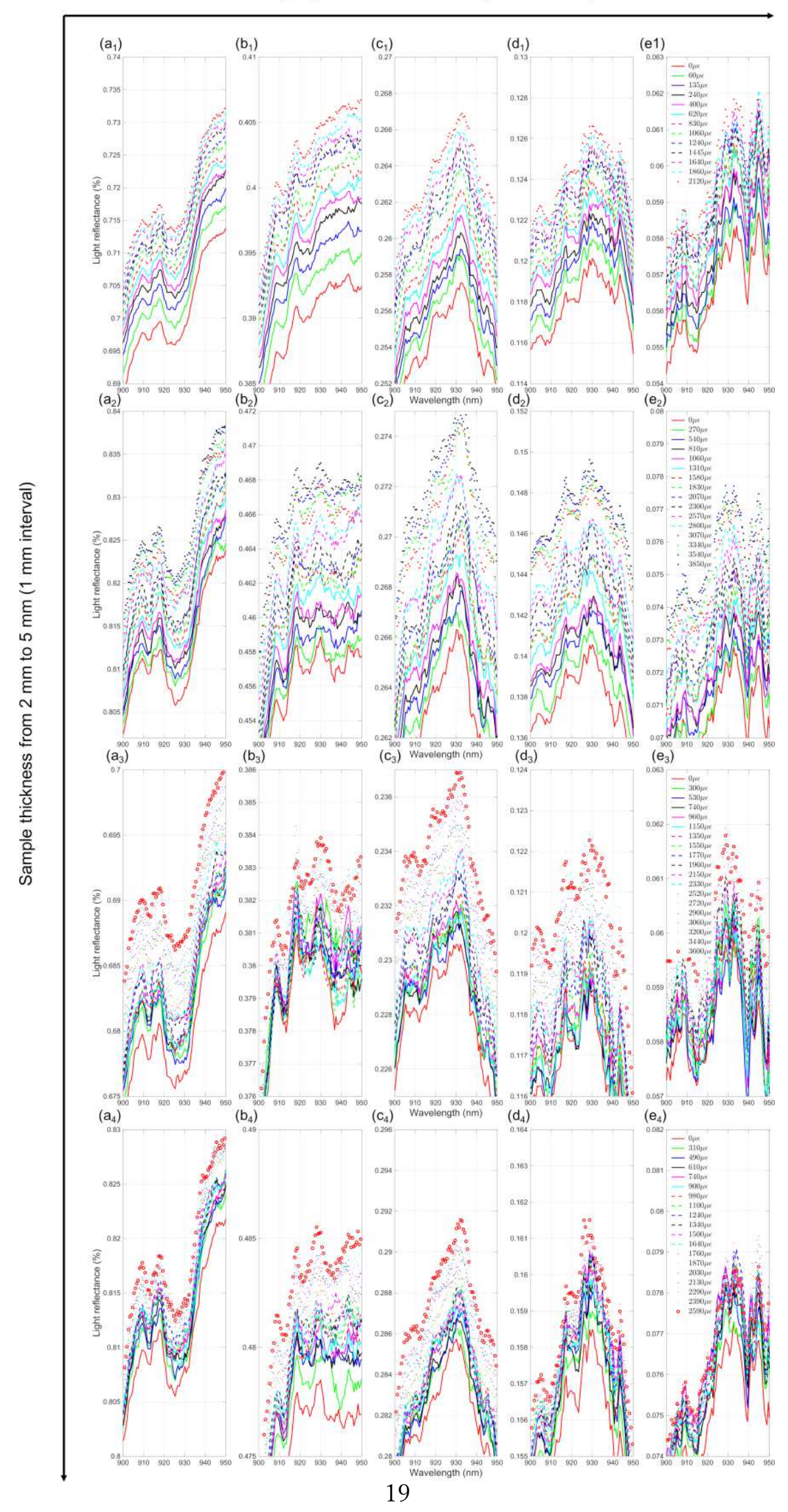


Fig. 4 Vis-NIR SRS spectral data collected at various sample thicknesses (vertical) by different fiber groups (horizontal).

Fig. 5 (b) and (c) show the digital photos of wood samples before and after tension

during the tension test.
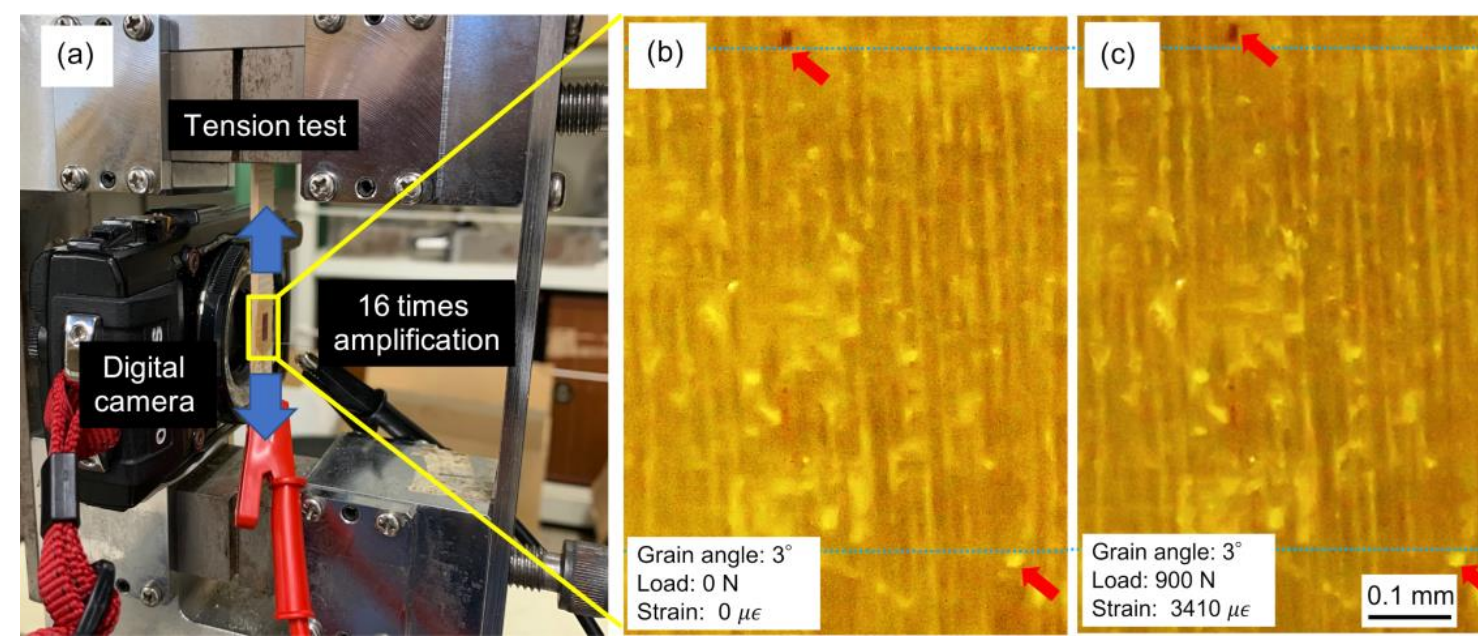

Fig. 5 Digital photos of the wood sample with $2 \mathrm{~mm}$ thickness before (a) and after (b) the tension test, respectively. 
307 accumulated contribution rate of the frist two scores is approximately $99.64 \%, 99.15 \%$,

$30897.47 \%$, and $94.48 \%$ for a sample thickness of $2,3,4$, and $5 \mathrm{~mm}$, respectively. The PC1

309 and PC2 scores of the SRS are shown in Fig. $6\left(c_{1-4}\right)$, where the Y-axis shows PC2 and

310 the X-axis shows the PC1. There is a strong correlation between PC1 loading and light

311 scattering differences, i.e., vertical baseline shift. As expected from Fig. 4, the

312 contribution rate of the PC 1 score decreases with an increase in sample thickness.

313 Moreover, PC2 loading has relatively high absolute values at a light wavelength of 970

$314 \mathrm{~nm}$. This suggests a meaningful correlation between the light absorption by hydrogen

315 bonds and wood strain changings, but the contribution rate was much lower than the light

316 scattering differences. 

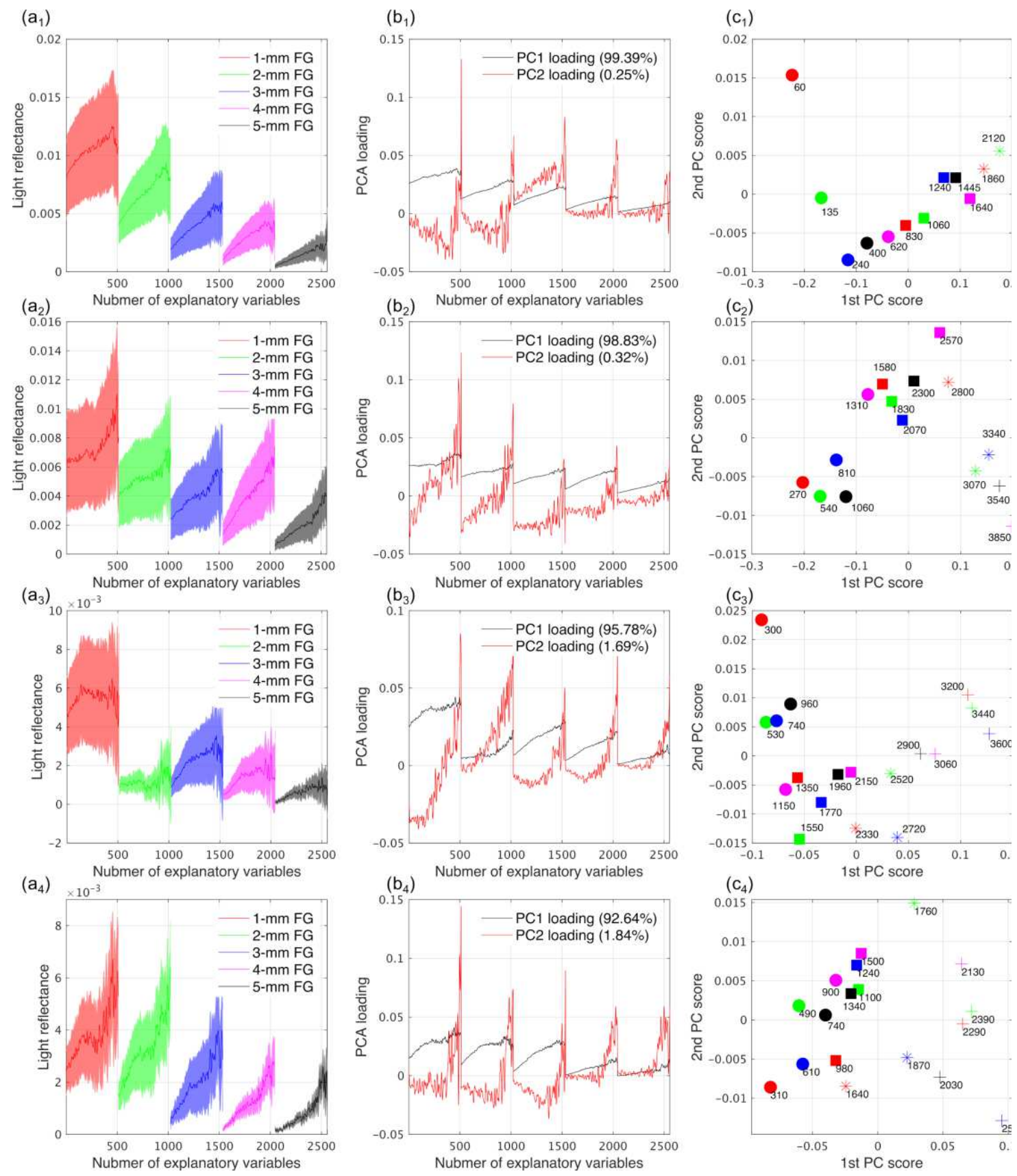

Fig. 6 (a1-4) Concatenated Vis-NIR difference spectral data used for PCA; (b1-4) first two principal component loadings; $\left(c_{1-4}\right)$ first two principla component scores of the concatenated Vis-NIR spectral data.

Fig. 7 (a) shows the strain calibration results of the wood samples with the same thickness $(2 \mathrm{~mm}$ ) from the PLS regression method with LV numbers 9 (explanatory 
variables: Vis-NIR difference spectral data after pretreatments; response variables: strain

321 reference values). The density of the samples was $415 \pm 14 \mathrm{~kg} / \mathrm{m}^{3}$ and the MC was

$32210.51 \% \pm 0.26 \%$. Overall, the PLS calibration model has a high prediction accuracy: the

$323 R^{2}$ and RMSE of the calibration set are 0.86 and $297.89 \mu \varepsilon$, respectively. For the validation

324 set, the $R^{2}$ and RMSE are 0.82 and $345.44 \mu \varepsilon$, respectively. Differences in the RMSE can

325 be attributed to the fact that the strain gauge only measured the surface strain of each

326 wood sample, whereas the SRS method measured the whole strain with the information

327 of sample thickness. Indeed, there is a possibility that the SRS method can more

328 accurately measure the wood sample strain than conventional strain gauges, but further

329 studies are required to prove this. Overall, this is the first study to suggest that the Vis-

330 NIR SRS method combined with PLS regression analysis can predict the tensile of wood

331 samples with the same thickness (also see the prediction results of the wood samples with

332 the same $5 \mathrm{~mm}$ thickness in supplementary, Fig. S2). Fig. 7 (b) shows the strain

333 calibration results when using the raw Vis-NIR spectral data, it is evident that the

334 difference spectral data could be used to achieve the initial value correction purpose, thus

335 are necessary to construct the strain calibration model. 
(a)

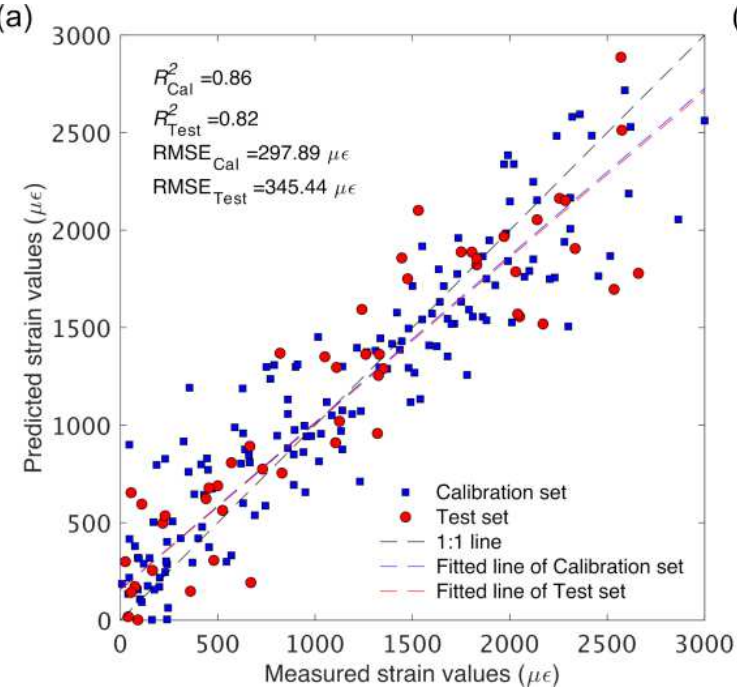

(b)

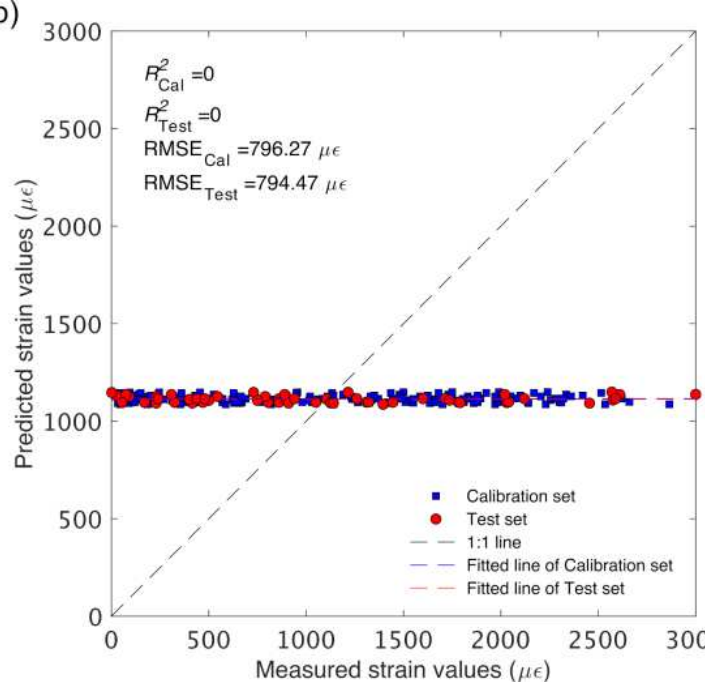

Fig. 7 Scatter plot of measured and predicted strain values using (a) difference spectral data or (b) raw spectral data.

Fig. 8 (a) shows the VIP scores, from which it is evident that the number of important

detection and illumination. Fig. 8(b) shows the PLS regression coefficients regarding

rate calculated by PCA at the wavelengths related to the hydrogen bonds is much lower

calibration model using all 18 samples. It could be supportted by the knowledge that wood also affects the light scattering degree (Konagaya et al. 2016). This also suggests that MC

MC may be the best way to reduce the MC effects. 
(a)

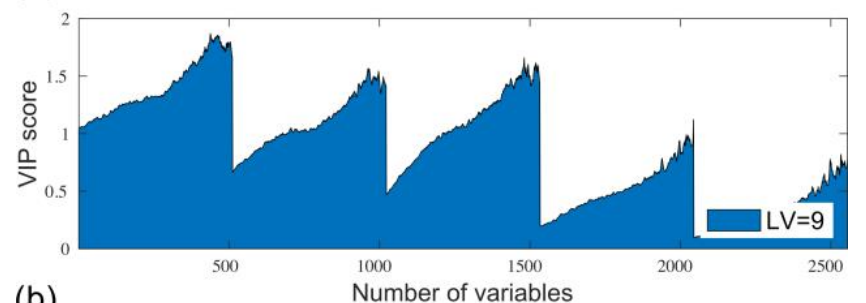

(b)

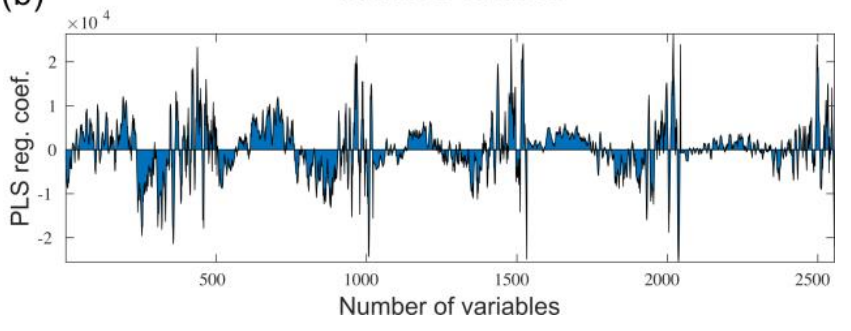

Fig. 8 (a) VIP scores of the SRS spectral data; (d) PLS regression coefficients.

\section{Conclusion}

This study aims to demonstrate the correlation between light scattering changes inside

the wood cell walls and tensile strain. Spatially resolved diffuse reflectance was collected by designing a portable and cost-effective measurement system based on fiber probes.

354 For the preliminary experiment, samples with different thicknesses $(2 \mathrm{~mm}, 3 \mathrm{~mm}, 4 \mathrm{~mm}$, and $5 \mathrm{~mm}$ ) were measured to evaluate the influence of thickness. Then, for the primary experiment, 18 wood samples with the same thickness $(2 \mathrm{~mm})$ were tested to construct a and an RMSE for $297.89 \mu \varepsilon$ for five-fold cross-validation, 0.82 and $345.44 \mu$ for test validation. The designed SRS measurement system does not require sophisticated measurement techniques. Moreover, it has a cost design effective design due to the Vis- 
361 NIR HSI camera with short-wave sensitivity, which is much cheaper than long-wave

362 sensitivity cameras.

363 Further research should focus on extending the applicability of the SRS approach to

364 a broader database of wood types and larger sample numbers with various thicknesses.

365 The intervals between fiber groups could be changed to test the strain predicion of thicker

366 wood samples. This research also references further research to measure growth strain in

367 trees non-destructively. However, because light scattering degree is also affected by MC,

368 this would require more in-depth spectral pretreatments.

\section{Acknowledgments}

371 The authors are grateful for the financial support provided by JSPS (KAKENHI, no.19K15886)

\section{References}

375 Adler DC, Buehler MJ (2013) Mesoscale mechanics of wood cell walls under axial strain. Soft Matter 9:7138-7144. https://doi.org/10.1039/c3sm50183c

377 Åkerholm M, Salmén L (2001) Interactions between wood polymers studied by dynamic FT-IR spectroscopy. Polymer (Guildf) 42:963-969. 
https://doi.org/10.1016/S0032-3861(00)00434-1

Altaner CM, Thomas LH, Fernandes AN, Jarvis MC (2014) How cellulose stretches: 798. https://doi.org/10.1021/bm401616n 
397 Cen H, Lu R (2010) Optimization of the hyperspectral imaging-based spatially-resolved system for measuring the optical properties of biological materials. Opt Express 18:17412. https://doi.org/10.1364/oe.18.017412

Cuesta Sánchez F, Toft J, van den Bogaert B, et al (1995) Monitoring powder blending https://doi.org/10.1007/BF00323062

D’Andrea C, Farina A, Comelli D, et al (2007) Time-resolved diffuse optical https://doi.org/10.1118/1.596777

Farrés M, Platikanov S, Tsakovski S, Tauler R (2015) Comparison of the variable

Farrell TJ, Patterson MS, Brain W (1992) A diffusion theory model of spatially resolved, steady-state diffuse reflectance for the noninvasive determination of tissue optical properties in vivo. Medphys 19:879-888.

\footnotetext{
Farrés M, Platikanov S, Tsakovski S, Tauler R (2015) Comparison of the variable
} importance in projection (VIP) and of the selectivity ratio (SR) methods for variable selection and interpretation. J Chemom 29:528-536. 
https://doi.org/10.1002/cem.2736

416 Gorry PA (1991) General Least-Squares Smoothing and Differentiation of https://doi.org/10.1021/ac00005a031

Guo F, Altaner CM, Jarvis MC (2020) Thickness-dependent stiffness of wood: Potential mechanisms and implications. Holzforschung 74:1079-1087. https://doi.org/10.1515/hf-2019-0311

Guo F, Cramer M, Altaner CM (2019) Evaluation of near infrared spectroscopy to nondestructively measure growth strain in trees. Cellulose 26:7663-7673. https://doi.org/10.1007/s10570-019-02627-2

Hon DNS, Chang ST (1984) Surface Degradation of Wood By Ultraviolet Light. J Polym Sci A1 22:2227-2241. https://doi.org/10.1002/pol.1984.170220923

Hsieh YC, Yano H, Nogi M, Eichhorn SJ (2008) An estimation of the Young's modulus of bacterial cellulose filaments. Cellulose 15:507-513. https://doi.org/10.1007/s10570-008-9206-8 
433 Kamiyama T, Suzuki H, Sugiyama J (2005) Studies of the structural change during deformation in Cryptomeria japonica by time-resolved synchrotron small-angle $\mathrm{X}$ ray scattering. J Struct Biol 151:1-11. https://doi.org/10.1016/j.jsb.2005.04.007 0273 
451 Ma T, Schajer G, Inagaki T, et al (2018a) Optical characteristics of Douglas fir at

452 various densities, grain directions and thicknesses investigated by near-infrared spatially resolved spectroscopy (NIR-SRS). Holzforschung 1-8. https://doi.org/10.1515/hf-2017-0213

Ma T, Schajer G, Inagaki T, et al (2018b) Optical characteristics of Douglas fir at various densities, grain directions and thicknesses investigated by near-infrared spatially resolved spectroscopy (NIR-SRS). Holzforschung 72:789-796. https://doi.org/10.1515/hf-2017-0213

Ma T, Tsuchikawa S, Inagaki T (2020) Rapid and non-destructive seed viability prediction using near-infrared hyperspectral imaging coupled with a deep learning approach. Comput Electron Agric 177:. https://doi.org/10.1016/j.compag.2020.105683

Martens H, Tormod N (1992) Multivariate calibration. John Wiley \& Sons.

Marthin O, Kristofer Gamstedt E (2019) Damage shielding mechanisms in hierarchical composites in nature with potential for design of tougher structural materials. $\mathrm{R}$ Soc Open Sci 6:. https://doi.org/10.1098/rsos.181733

Mohammadi-Moghaddam T, Razavi SMA, Sazgarnia A, Taghizadeh M (2018) Predicting the moisture content and textural characteristics of roasted pistachio 
Montero C, Clair B, Alméras T, et al (2012) Relationship between wood elastic strain under bending and cellulose crystal strain. Compos Sci Technol 72:175-181. https://doi.org/10.1016/j.compscitech.2011.10.014

Mvondo RRN, Meukam P, Jeong J, et al (2017) Influence of water content on the mechanical and chemical properties of tropical wood species. Results Phys 7:2096-2103. https://doi.org/10.1016/j.rinp.2017.06.025

Okazaki Y (2012) Near-Infrared Spectroscopy-Its Versatility in Analytical. Anal Chem 28:545-562

Ozyhar T, Hering S, Niemz P (2012) Moisture-dependent elastic and strength anisotropy of European beech wood in tension. J Mater Sci 47:6141-6150. https://doi.org/10.1007/s10853-012-6534-8

Peng Y, Lu R (2008) Analysis of spatially resolved hyperspectral scattering images for assessing apple fruit firmness and soluble solids content. Postharvest Biol Technol 48:52-62. https://doi.org/10.1016/j.postharvbio.2007.09.019 cellulose in Norway spruce. Wood Sci Technol 41:565-583. 
https://doi.org/10.1007/s00226-007-0141-x

488

489

490

491

492

493

494

495

496

497

498

499

500

501

502

503

504

Qin J, Lu R (2008) Measurement of the optical properties of fruits and vegetables using spatially resolved hyperspectral diffuse reflectance imaging technique. Postharvest Biol Technol 49:355-365. https://doi.org/10.1016/j.postharvbio.2008.03.010

Qin J, Lu R, Peng Y (2009) Prediction of apple internal quality using spectral absorption and scattering properties. Trans ASABE 52:486-499. https://doi.org/10.13031/2013.26807

Salmén L (2015) Wood morphology and properties from molecular perspectives. Ann For Sci 72:679-684. https://doi.org/10.1007/s13595-014-0403-3

Salmén L, Bergström E (2009) Cellulose structural arrangement in relation to spectral changes in tensile loading FTIR. Cellulose 16:975-982. https://doi.org/10.1007/s10570-009-9331-z

Smith I, Landis E, Gong M (2003) Fracture and fatigue in wood. John Wiley \& Sons

Tkachenko N V (2006) Chapter 7 - Flash-photolysis. In: Optical Spectroscopy. Elsevier Science, Amsterdam, pp 129-149

Vanoli M, Van Beers R, Sadar N, et al (2020) Time- and spatially-resolved spectroscopy to determine the bulk optical properties of 'Braeburn' apples after ripening in shelf life. Postharvest Biol Technol 168:. 
https://doi.org/10.1016/j.postharvbio.2020.111233

506 Wold S, Johansson E, Cocchi M (1993) PLS: partial least squares projections to latent structures Quantification of Acidity of Aviation Kerosene. Open Fuels Energy Sci J 1:51-53. surface strain in trees: A review. Aust For 68:34-43. https://doi.org/10.1080/00049158.2005.10676224

Yu Y, Jiang Z, Tian G (2009) Size effect on longitudinal MOE of microtomed wood https://doi.org/10.1007/s11632-009-0040-3

Zhu Q, He C, Lu R, et al (2015) Ripeness evaluation of "Sun Bright” tomato using optical absorption and scattering properties. Postharvest Biol Technol 103:27-34. https://doi.org/10.1016/j.postharvbio.2015.02.007

Zude M, Pflanz M, Spinelli L, et al (2011) Non-destructive analysis of anthocyanins in cherries by means of Lambert-Beer and multivariate regression based on spectroscopy and scatter correction using time-resolved analysis. J Food Eng 


\section{Supplementary Files}

This is a list of supplementary files associated with this preprint. Click to download.

- Supplementary20210524.pdf 